\title{
Lessons Learned from Using Health Literacy Strategies in a Pilot Communication Skills Program
}

\author{
Rosina A. Connelly ${ }^{*}$, , Teri L. Turner ${ }^{2}$, Xuan G. Tran ${ }^{3}$ and Angelo P. Giardino ${ }^{2}$ \\ ${ }^{I}$ Department of Pediatrics and Adolescent Medicine, University of South Alabama-Mobile, AL, USA \\ ${ }^{2}$ Department of Pediatrics, Section of Academic General Pediatrics, Baylor College of Medicine-Houston, TX, USA \\ ${ }^{3}$ Health Services Research and Continuing Medical Education, Texas Children's Health Plan-Houston, TX, USA
}

\begin{abstract}
Introduction: Limited health literacy results in poorer health outcomes, however, effective communication can facilitate understanding. Communication skills programs could incorporate strategies to address communication gaps caused by poor health literacy.

Objectives: 1) to describe the effects of a pilot educational intervention on providers' knowledge and reported use of health literacy strategies; 2) to describe participants' reasons to participate and their opinions regarding the educational intervention's delivery and content.

Methodology: We conducted a quasi-experimental study design with a questionnaire before, immediately after, one and three months after the intervention. Semi-structured interviews conducted one year after the intervention explored participants' opinions and experiences with the intervention and strategies.

Results: Of 329 physicians invited, only 13 (3.9\%) participated. Participants' mean knowledge score increased from $59.2 \%$ to $80 \%(\mathrm{p}<0.001)$ but was lower at three months $(63.3, \mathrm{p}<0.005)$. Reported awareness of health literacy issues increased from $23.1 \%$ to $92.3 \%(\mathrm{p}<0.001)$ and remained high at three months. Using simple language, limiting amount of information and checking for understanding were strategies reportedly still used at three months. Information presented was new for participants and increased their awareness of communication problems. Health literacy strategies were reportedly simple to use.

Conclusions: Our program increased participants' awareness of health literacy issues and self-reported use of health literacy strategies for communication up to three months after the intervention. Future research areas should include replication with a larger sample size, objective measurement of strategies utilized by providers, and measuring patients' opinions about these strategies.
\end{abstract}

Keywords: Health literacy, communication skills, continuing medical education.

\section{INTRODUCTION}

The National Institutes of Health defines health literacy as the "degree to which individuals have the capacity to obtain, process and understand basic health information and services needed to make appropriate health decisions" [1]. This concept has been under scrutiny as causal pathways for health literacy and associated health outcomes continue to be elucidated [2]. The 2005 report from the National Center for Education Statistics reported that half of American adults or 90 million people in the United States had limited health literacy skills $[1,3]$. Though low health literacy could affect anyone, racial and ethnic minorities, recent immigrants, persons of low socioeconomic status, and the elderly are significantly more affected $[4,5]$.

Health literacy affects communication and interaction between patients and providers [2,6]. Poor communication

*Address correspondence to this author at the University of South Alabama, Department of Pediatrics and Adolescent Medicine, Division of General Pediatrics, 1504 Springhill Avenue, Room 5316, Mobile, AL 36604, USA; Tel: (251) 434-3971; Fax: (251) 434-3980;

E-mail: rosinaconnelly@usouthal.edu may result in medication errors, lack of compliance, increased healthcare costs, patient dissatisfaction, physician frustration, and higher rates of malpractice claims [7-9]. A national survey by Turner et al. showed that pediatricians reportedly were aware of communication gaps [10]. Even when almost all pediatricians in this survey reportedly used simple language as a communication strategy, studies with more objective evaluation of conversations during clinical encounters have found that health professionals often use jargon and do not check for understanding when communicating with patients [11]. While it would be difficult to change patients' overall health literacy skills, healthcare providers could learn how to employ strategies designed to address low health literacy, which may improve communication with all patients and positively affect health outcomes [6].

We hypothesized that an educational intervention on health literacy strategies for communication would result in increased knowledge of the health literacy problem, as well as increased frequency of use of health literacy strategies for communication by pediatric healthcare providers. The objectives of this study were: 1) to describe the effects of a 
pilot educational intervention on providers' knowledge and reported use of health literacy strategies; and 2) to describe participants' reasons to participate and their opinions regarding the educational intervention's delivery and content.

\section{METHODOLOGY}

This was a mixed-methods analysis of quantitative and qualitative data. A quasi-experimental study design was used to assess participants' reactions and learning from the communication skills training program with a questionnaire used before, immediately after, at one month, and three months after the educational intervention. Semi-structured interviews were conducted one year after the intervention to explore participants' opinions about the intervention and the strategies.

\section{Intervention}

We developed a focused educational intervention consisting of a 2-hour workshop for pediatric health care providers. The workshop included a didactic session, the showing of a video clip, group discussion and role-playing using a common clinical scenario. The short didactic session included an overview of the health literacy problem in the United States, its effects on health, and its role as a potential barrier to effective health communication. The video from the American Medical Association (AMA) Foundation's educational tool 'Health Literacy: Help Your Patients Understand' [4] portrayed actual patients describing how their experiences during medical situations and miscommunications with physicians had negatively affected their health. Participants were introduced to health literacy strategies for improved communication that are available from this AMA's educational tool (e.g. checking for patient's understanding, avoiding medical jargon, encouraging patients to ask questions, speaking slowly, and limiting the amount of information) [4] and from the Partnership for Clear Health Communication's 'Ask Me 3 TM' questions: 1. What is my [child's] main problem? 2. What do I need to do? 3. Why is it important for me to do this? [12].

Three interactive exercises: a case scenario, an active exercise and role play activity were used to practice communication using the health literacy strategies presented. During the case scenario, participants identified potential communication barriers during the hypothetical encounter presented. For the active exercise, participants translated selected medical jargon words into plain language. For the role play exercise, participants portrayed a patient, doctor, or observer/reporter during a hypothetical clinical scenario utilizing the health literacy strategies they learned during the didactic session.

\section{Population/Setting}

Two groups of pediatric providers were invited to participate. The first group included providers from 80 of the highest patient volume offices in the network for Texas Children's Health Plan (TCHP), a provider-owned pediatric HMO affiliated with Texas Children's Hospital (TCH), serving approximately 130,000 enrollees with Medicaid and State Children's Health Insurance Program. The second group consisted of community physicians employed by Texas Children's Pediatric Associates (TCPA), a large multi- site primary care practice group entirely owned by $\mathrm{TCH}$, which provides pediatric care to approximately $20 \%$ of children in the Houston metropolitan area. Participants could choose from three workshop date/time options: a Saturday morning, a weekday morning, and a weekday evening. The date/time with most responses was selected for workshop offering, and reminders were sent the week prior to the workshop.

To encourage TCHP providers' participation in the educational intervention incentives offered included provision of 2 hours of AMA Category 1 CME credits, a $\$ 100$ honorarium, validated parking, and refreshments during the workshop. All participating providers were offered an additional $\$ 50$ for participating in the phone interviews one year after the intervention. Although TCPA providers were not offered an honorarium for workshop attendance, the other incentives remained the same.

\section{Data Collection/Analysis}

A questionnaire with items from the AMA Foundation's educational tool 'Health Literacy: Help Your Patients Understand' [4] included multiple choice and true/false items to measure health literacy knowledge and was scored based on the number of correct answers. Likert scale questions were used to measure providers' report of behaviors they used to facilitate doctor-patient communication in their practices (i.e. use of 'Ask Me $3^{\mathrm{TM}}$ ' questions, or the teach-back technique). Quantitative information was collected immediately before and after the educational intervention. Follow up questionnaires were administered one and three months after the intervention. Data regarding knowledge of health literacy issues and reported use of health literacy strategies for communication were analyzed using descriptive statistics, paired t-test, and Wilcoxon signed-rank test when applicable. Using the statistical software STATA version 9.0 for sample size and power calculations, assuming a power of 0.8 , alpha of 0.05 , and attrition rate of $20 \%$ at 3 months, we anticipated that with regard to knowledge of health literacy issues 18 subjects were needed to detect a $50 \%$ difference in test scores. For reported use of health literacy strategies, it was estimated that a sample size of 45 subjects was needed to detect a $10 \%$ increase in number of pediatric health care providers reporting use health literacy strategies for communication most of the times or always after the educational intervention.

One year after the initial educational intervention, we conducted semi-structured interviews for the qualitative portion of the study. All providers who attended the workshop offerings were invited to participate in the follow up phone interviews. Phone conversations with those who volunteered to participate were recorded without identifiers and subsequently transcribed by a third party. Two of the investigators ( $\mathrm{RC}$ and $\mathrm{AG}$ ) analyzed interview data for content using the constant comparison method to identify recurrent themes. After cross checking for content analysis, these two authors summarized and arrived at consensus of the themes presented.

This protocol was reviewed and approved by the Institutional Review Board at Baylor College of Medicine. 


\section{RESULTS}

\section{Participants' Characteristics}

Three hundred twenty-nine providers were invited to participate. Of these, only $18(5.6 \%)$ replied to the invitations, $14(4.3 \%)$ attended the workshop, and $13(3.9 \%)$ completed pre and post intervention questionnaires and agreed to complete the follow up questionnaires. Of all participants, $9(69.2 \%)$ were female, $12(92.3 \%)$ were pediatricians, and $1(7.7 \%)$ was a family medicine physician. Mean participants' age was 56.6 years (range 39 - 70); and mean number of years in practice was 23.8 (range 11-33). None of the providers had participated in previous communication skill training programs, although one provider later reported having read the AMA Foundation's educational tool 'Health Literacy: Help Your Patients Understand' [4]. There was no information available on the providers who received invitations but chose not to participate in the educational workshops.

\section{Quantitative Analysis}

\section{Effects on Participants' Health Literacy Knowledge}

There were 10 items in the questionnaires covering facts about low literacy and the effects of low literacy on health. The mean score was $59.2 \%$ (SD 14.4) prior to the educational intervention and increased to $80 \%$ (SD 10.4) immediately after the intervention, with a significant difference between the two means using paired t-test analysis $(\mathrm{p}=0.0004)$.

\section{Effects on Awareness of Health Literacy Problem}

Prior to the educational intervention only 3 (23.1\%) participants reported being 'very aware' of the health literacy problem, while $12(92.3 \%)$ participants reported being 'very aware' of the problem after the educational intervention. Comparison of these level of awareness before and after the intervention done using Wilcoxon signed-rank test showed a significant increase in the level of awareness of the health literacy problem immediately after the intervention $(\mathrm{p}=0.003, \mathrm{Z}=-2.954)$.

\section{Participants' Reported Use of Health Literacy Strategies}

Participants reported their frequency of use of specific health literacy strategies for communication during the week prior to the intervention, as well as their intention to use those strategies during the week following the educational intervention. This information by specific health literacy strategies is presented in Table 1. Using Wilcoxon signedrank test, the number of strategies that providers intended to use 'most of the time' or 'always' immediately after the educational intervention significantly increased compared to their reported use before the intervention $(p=0.001, Z=-$ 3.18).

\section{One and Three-Month Follow Up}

A total of 7 (53.8\%) participants returned the one-month follow up questionnaire, while $6(46.1 \%)$ returned the threemonth follow up questionnaires. Mean knowledge score at one and three-month after the intervention were $71.5 \%$ (SD15.7) and 63.3\% (SD 10.3) respectively. Seven out of original $13(53.8 \%)$ providers reported to be 'very aware' of the health literacy issues at one month follow up, and 5 out of $13(38.5 \%)$ three months after the intervention. The information on health literacy strategies reportedly used 'most of the time' or 'always' at one and three months after the intervention is presented in Table 2.

\section{Qualitative Analysis}

All 13 providers who participated in the educational workshops were invited to participate in follow up phone interviews one year after the intervention. Seven $(53.8 \%)$ providers volunteered to participate, and only $4(31 \%$ response rate) completed the follow up phone interviews. The other 3 providers who agreed to participate could not be reached by telephone after three attempts on the scheduled date/time for interview. A summary of the themes, based on participants' responses is presented in Table $\mathbf{3}$.

\section{Excerpts of Interviews}

In discussing the reasons for participating in the communication skills workshop participants highlighted their desire to learn more effective communication skills, due to the realization that there was miscommunication with patients. Some responses to the question about reasons to participate are shown:

Participant 1: Because at the time it sounded like I could get a lot out of it. Because most of the time we think, "Oh we're doing a great job of communicating with your patients," and then when you go back or the patient comes back and you ask them something and, all the sudden you realize they didn't understand anything you said... I find some patients are very easy to communicate with... Other

Table 1. Reported Use of Health Literacy Strategies Before, Compared to Intention to Use After the Intervention

\begin{tabular}{|c|c|c|c|}
\hline "Communication Techniques that I have Used in the Past Week/Will Use in the Next Week" & $\begin{array}{l}\text { Pretest } \\
(n=13) \\
N(\%)\end{array}$ & $\begin{array}{c}\begin{array}{c}\text { Posttest } \\
(n=13)\end{array} \\
\text { N (\%) }\end{array}$ & p Value \\
\hline Asking patients to repeat information, by "teach back" or "show me" technique & $3(23.1)$ & $10(76.9)$ & 0.008 \\
\hline Presenting 2 or 3 concepts at a time and checking for understanding & $7(53.8)$ & $12(92.3)$ & 0.025 \\
\hline Using simple language (avoid technical jargon) & $11(84.6)$ & $13(100)$ & 0.15 \\
\hline Using models to explain & $1(7.7)$ & $8(61.5)$ & 0.01 \\
\hline Using Ask Me 3 Questions & 0 & $10(76.9)$ & 0.01 \\
\hline
\end{tabular}


Table 2. Reported Use of Health Literacy Strategies One and Three-Month Follow Up After the Intervention

\begin{tabular}{|c|c|c|}
\hline "Communication Techniques that I have Used in the Past Week" & $\begin{array}{c}\text { One-Month } \\
(\mathbf{n}=7) \\
\mathbf{N}(\%)\end{array}$ & $\begin{array}{c}\text { Three-Month } \\
(\mathbf{n}=\mathbf{6}) \\
\text { N (\%) }\end{array}$ \\
\hline Presenting 2 or 3 concepts at a time and checking for understanding & $6(85.7)$ & $4(66.7)$ \\
\hline Using simple language (avoid technical jargon) & $7(100)$ & $5(83.3)$ \\
\hline Using models to explain & $1(14.3)$ & $2(33.3)$ \\
\hline Using Ask Me 3 Questions & $1(14.3)$ & $4(66.7)$ \\
\hline
\end{tabular}

Table 3. Summary of the Themes, Based on Participants' Responses

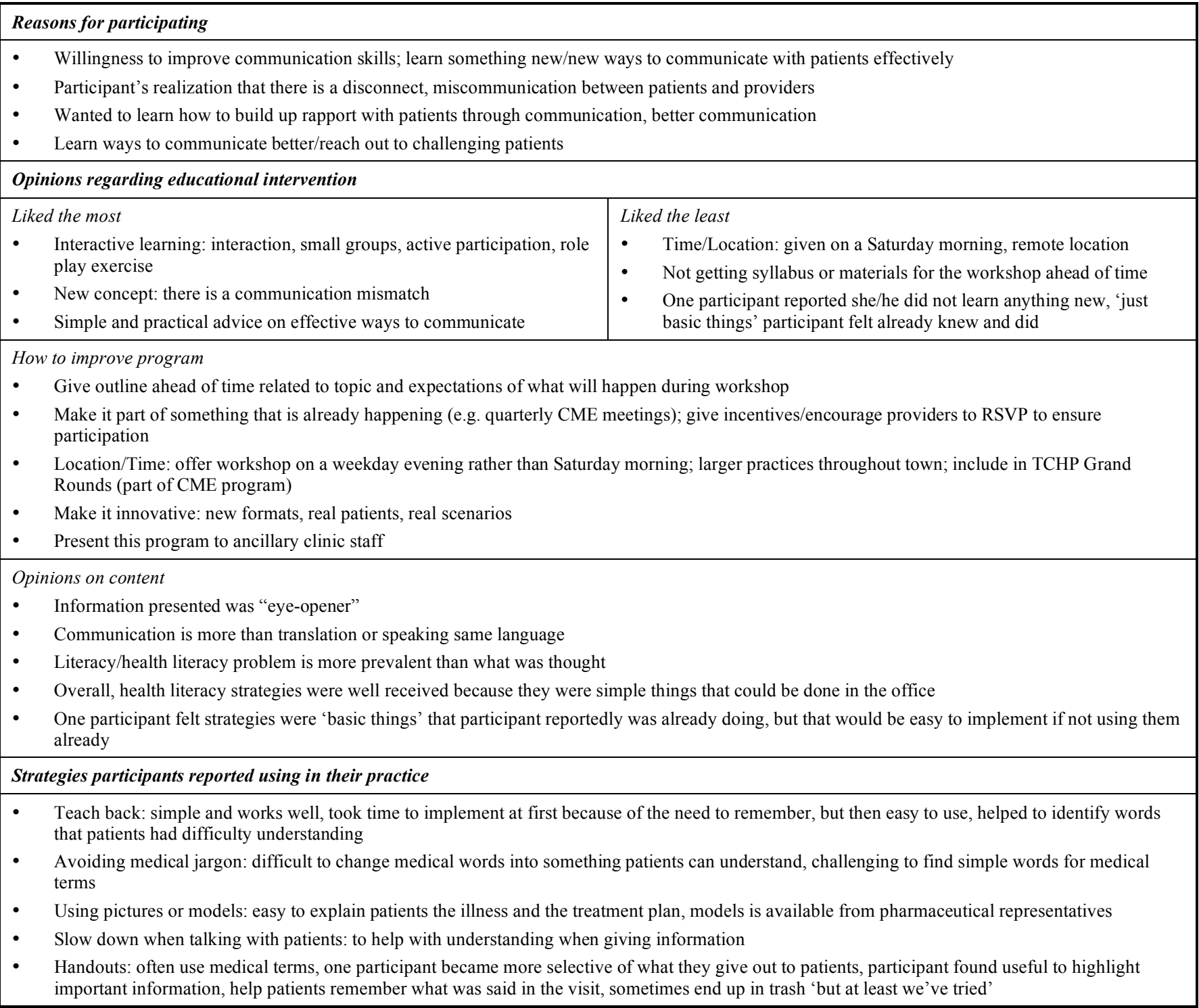

patients, you never get a feel for what it is that they don't understand. They never tell you, "Oh okay, I didn't get, you know, what you are trying to tell me." And so those are the patients that I think I was more concerned about trying to learn something to get to those patients.

Participant 2: Sometimes after I've talked to the patient, they look at me, say, "yes, yes, yes". "Do you have any questions?" They say 
"No, no, no". And when I leave the room then here comes the medical assistant who says, "The patient in room so and so wants to ask you some more questions." And so, obviously at the time I really had some lack of confidence in...feeling that the patient understood what I said...I needed to find out how I could interview them in a better way.

Participants were asked to express their opinion about the program, the usefulness of the information presented, the format, what they liked most, and what they liked the least. Some excerpts illustrate their thoughts:

Participant 1: ... sometimes we're practicing medicine so fast and I mean, I don't know about some of my colleagues, but I find that my office staff and myself, sometimes we're like chasing each other... And sometimes I'll even say, "Oh, did I even answer the patient correctly?" And so, going to something like this...made us stop and re-evaluate what we were doing. And the second thing, it gave us some easy things that we could do in our office. It wasn't like you asked me to go out and buy an MRI machine... It was a simple, "Ask the patient, did they understand?" Have them come back to you and explain what you just told them. Or something as simple as [using] a bunch of models... [Then talking about the workshop format]...Probably that it was a really small group... so that you didn't feel like you were going to ask the wrong question.

Participant 2: [talking about the role play activity]...where one doctor acted as the patient, the other doctor acted as the doctor... I like the interactions.

Participant 4: I think everything was fine. It was just that I thought maybe there will be some specific guidelines which I didn't' get, you know. They were just average rules and regulations of how to deal with patients and how to get the proper history and communicate with the family and all that, but nothing specific. These are basic things that we already do.

In response to the actual content presented during the workshop, participants expressed that the information presented, which focused on the amount of miscommunication that occurs during health care interactions due to low health literacy, was an 'eye opener'. Four excerpts related to this theme are shown below:

Participant 1: Because it's such a new concept, I mean I think we all went to medical school and said, "Okay, you can now, you know, tell anybody anything, and they are all going to understand." Nobody ever told us everybody is on a different level of understanding... I was talking to another physician who is not a pediatrician and he said, "All the sudden you realize you don't walk on water." I said, "Yeah."... I said, "It was very, very shocking."

Interviewer: So you commented about the workshop to another physician.

Participant: $\mathrm{Mm}-\mathrm{hmm}$.

Interviewer: who is not in Pediatrics?

Participant: No, he's in Internal Medicine, and we were talking, and I was asking him, I said, "Do you sometimes feel that your patients don't understand what you are talking about?" And of course, like all of us, we say, "Most of my patients know what I'm talking about. I talk at their level." And so I asked him to look, and then we got together, because we're longtime friends... And I said, "Well, how was it?" And he goes, "Well, I don't walk on water either."

Participant 2: Oh yes, I remember but it's such an overwhelming huge problem, you know...It's not just English and Spanish, you know.

Participant 3: Well, one of things that I recall is that illiteracy in general was much more prevalent than most of us cared to realize, and when it came specifically to medical issues, yes, it's even greater.

Finally, in response to a question about the actual health literacy strategies discussed during the workshop and their experience with using these in practice:

Participant 1: It was probably hard to incorporate it because I would have to remember...so at first, it did take a little longer, but then I think my patients taught me some of the stuff, some of the words, again I think it's the words that the patients confuse or the patients don't understand. You know, what words were good, what words were not so good, and then what words were bad, I mean as far as the patient... And for them to walk away and say, "Now, what did I have?"

Participant 3: Well, I remember some of the things that ya'll talked about I was already doing. One is to provide handouts in a semireadable format... We do sometimes find them in the trash, but at least we've tried. Sometimes I slow down a little more as far as how quickly I talk.

Participant 4: We made the language as simple and as everyday as possible, and we do that. We already [did] that. In fact, I never use complicated terms even with the educated patients...

\section{DISCUSSION}

Healthcare providers' ability to convey information in ways that patients can understand is essential for effective health communication and is one of the six objectives in the Health Communication Focus Area from the United States 
Healthy People 2010 [13, 14]. Furthermore, the American Medical Association Council report on Health literacy has recommended 'the development of undergraduate, graduate, and continuing medical education programs that train physicians to communicate with patients who have limited literacy skills' [13].

A systematic review by Davis et al. about continuing medical education (CME) programs found that learnercentered, interactive, engaging, and reinforcing programs were more effective in change physicians' behavior, and occasionally positively impacted health outcomes [15]. And although short educational programs have been found to have no effect on changing physicians' behavior [16], research in the area of communication skills training has shown that programs focused on specific skills were likely to be effective in changing physicians' behavior [17]. A study by Shilling et al. revealed that patients reported more satisfaction after providers' participation in a communication skills training [18]. In another study, physicians who used specific communication behaviors (i.e. orienting statements, checking for understanding) were less likely to be involved in malpractice claims [8]. Communication skills training is often used by organizations as a mean to ultimately improve overall patient satisfaction [19]. Long-term results of these programs, nevertheless, seem to depend on the length and quality of the program $[17,19]$.

At the time of our literature review, there were no published studies regarding communication programs that specifically used health literacy strategies and targeted pediatric healthcare providers. Mika et al. were conducting and subsequently published a study using the Ask Me 3 strategy in a pediatric clinical setting, but their intervention did not involve detailed training of healthcare providers on this specific strategy [20].

Our study served as a pilot test for the design, content, implementation, and effectiveness of short educational intervention using health literacy strategies and intended to improve pediatric healthcare providers' communication skills. The overall goal is to develop an effective program for improved communication skills and ultimately improve patient satisfaction in the near future.

There were several limitations affecting our ability to measure our outcomes. Low participation rates despite efforts for advertising, incentives, and reminders to promote attendance. This particular educational program did not have the popularity experienced by more scientific content traditional quarterly CME lectures offerings from the TCHP, with attendance to our workshop being lower than what we anticipated. While the actual delivery of our intervention was different from the traditional quarterly CME offerings from the TCHP, incentives and provision of CME credits were similar. After the qualitative portion of our study, we learned that attendance could potentially have been higher if the communication skills training were offered during the week, or as part of a more scientific topic which usually draws attention from community practitioners (e.g. mental health, dermatology, ophthalmology).

Through the qualitative portion of our analysis, we learned that providers had specific internal motivations to attend, including the pre-existing realization that there is a miscommunication with patients, and the desire to learn innovative ways to improve communication to positively affect health outcomes. These internal motivations, inherent to the desire to become a better physician, might have affected our ability to measure the effects of the intervention due to selection bias. Hence, providers with higher degree of internal motivation might have been more likely to participate and be ready to adopt the use of health literacy strategies beyond the incentives offered for participating in the educational program. Another limitation was the selfreported nature of the data, as participants answered questionnaires and provided a subjective measure of their own communication practices, which introduced potential information bias.

Overall, the educational intervention was well received and participants felt the information presented was an eye opener to the emergent issue of health literacy in health communication. Participants expressed benefiting from the interactive nature of the presentation. The excerpts of the AMA video presented were compelling and provided a deeper insight into the experiences of patients who have limited health literacy skills. The health literacy strategies were simple, practical and easy to use. One participant's experience with 'Teach Back' illustrated how it was helpful to identify specific words that were difficult to understand for subsequent use as part of the 'Using simple language' strategy. Another participant expressed disappointment with the educational program due to the perception that the strategies presented were 'basic things' the participant was already doing. This participant's report is consistent with findings of the survey done by Turner et al, where $99 \%$ of pediatricians reportedly used simple language when communicating with patients [10]. However, only $23 \%$ reportedly used teach-back to confirm understanding in this survey and other studies, which have also shown that physicians mostly use technical jargon [9-11, 21].

Our educational intervention increased participants' awareness of health literacy issues, and this effect persisted over time. With regards to the health literacy strategies presented, most participants had significantly high intentions to use these strategies for communication immediately after the intervention. Over time, the strategies participants reportedly continued to use were limiting information, checking for understanding, using simple language, and speaking more slowly. Specific strategies to assure understanding such as asking patients to repeat information and encouraging patients to ask questions (or using the Ask Me 3 questions) were reportedly used with less frequency. These findings were consistent with prior results by Farrell et al. and Schwartzberg et al. [5, 21].

Healthcare providers may not be aware of their own communication skills, as patients perceive them. As health communication continues to become an important measure of quality care and maintenance of certification, clinicians may change opinions regarding the need to assess and continuously improve their communication skills, and may be more willing to participate in this type of educational offerings. Even in the times of electronic medical records and telemedicine, parents of pediatric patients still expect that healthcare providers call them by their name and shake hands upon introductions [22]. Accordingly, the simple 
'basic' techniques utilized in our pilot communication skills program should be promoted for all clinicians to use in their patient-provider interactions to ultimately improve health outcomes [6].

\section{CONCLUSION}

The results of this mixed-methods analysis suggest that our pilot educational intervention was effective in increasing participants' knowledge and awareness of health literacy issues immediately after the intervention. Long-term effects on knowledge of and use of health literacy strategies for communication could not be measured to levels of statistical significance due to low participation and high attrition rates. Those who participated expressed inherent interest in improving communication skills to positively affect relationships with patients and health outcomes. Overall, health literacy strategies were well received due to their simplicity. Future research areas should include replication with a larger sample size, objective measurement of strategies utilized by providers, measuring patients' opinions about these health communication strategies, investigation of which strategy would be more effective in different clinical settings, and how the use of strategies affect health outcomes. Further research should also explore motivational factors to participate, as well as examine potential ways to increase importance of this topic to encourage clinicians' participation in this type of educational offerings.

\section{REFERENCES}

[1] Institute of Medicine. Health Literacy: A Prescription to End Confusion. Washington, DC: National Academies Press 2004.

[2] Paasche-Orlow M, Wolf M. The causal pathways linking health literacy to health outcomes. Am J Health Behav 2007; 31(Suppl 1): S19-S26.

[3] Rudd R. Health literacy skills of U.S. adults. Am J Health Behav 2007; 31(Suppl 1): S8-18.

[4] Weiss B. Health Literacy: a Manual for Clinicians. Chicago, IL: American Medical Association 2003.

[5] Schwartzberg J, Cowett A, VanGeest J, Wolf M. Communication techniques for patients with low health literacy: a survey of physicians, nurses, and pharmacists. Am J Health Behav 2007; 31 (Suppl 1): S96-S104.

[6] Hironaka L, Paasche-Orlow M. The implications of health literacy on patient-provider communication. Arch Dis Child 2008; 93(5): $428-32$.
[7] Levinson W, Stiles W, Inui T, Engle R. Physician frustration in communicating with patients. Med Care 1993; 31(4): 285-95.

[8] Levinson W, Roter D, Mullooly J, Dull V, Frankel R. Physicianpatient communication. The relationship with malpractice claims among primary care physicians and surgeons. JAMA 1997; 277(7): 553-9.

[9] Castro C, Wilson C, Wang F, Schillinger D. Babel Babble: Physicians' Use of Unclarified Medical Jargon with Patients. Am J Health Behav 2007; 31(Suppl 1): S85-S95.

[10] Turner T, Cull W, Bayldon B, et al. Pediatricians and health literacy: descriptive results from a national survey. Pediatrics 2009; 124(Suppl 3): S299-305.

[11] Lamiani G, Meyer E, Browning D, Brodsky D, Todres I. Analysis of enacted difficult conversations in neonatal intensive care. J Perinatol 2009; 29(4): 310-6.

[12] National Patient Safety Foundation PfCHC. Ask Me $3^{\text {TM }}$ Partnership for Clear Heatlh Communication; 2004 [cited 2009 04/01]; Available from: http: //www.npsf.org/askme3/.

[13] Health literacy: report of the Council on Scientific Affairs. Ad Hoc Committee on Health Literacy for the Council on Scientific Affairs, American Medical Association. JAMA 1999; 281(6): 552-7.

[14] USA Department of Health \& Human Services, Office of Disease Prevention and Health Promotion. Communicating Health: Priorities and Strategies for Progress. 2003 [cited 2009 7/15]; Available from: http: //www.odphp.osophs.dhhs.gov/projects/Hea lthComm/objective6.htm\#table4.

[15] Davis D, O'Brien M, Freemantle N, Wolf F, Mazmanian P, TaylorVaisey A. Impact of formal continuing medical education: do conferences, workshops, rounds, and other traditional continuing education activities change physician behavior or health care outcomes? JAMA 1999; 282(9): 867-74.

[16] Davis D, Thomson M, Oxman A, Haynes R. Changing physician performance. A systematic review of the effect of continuing medical education strategies. JAMA 1995; 274(9): 700-5.

[17] Levinson W, Roter D. The effects of two continuing medical education programs on communication skills of practicing primary care physicians. J Gen Intern Med 1993; 8(6): 318-24.

[18] Shilling V, Jenkins V, Fallowfield L. Factors affecting patient and clinician satisfaction with the clinical consultation: can communication skills training for clinicians improve satisfaction? Psychooncology 2003; 12(6): 599-611.

[19] Brown J, Boles M, Mullooly J, Levinson W. Effect of clinician communication skills training on patient satisfaction. A randomized, controlled trial. Ann Intern Med 1999; 131(11): 822-9.

[20] Mika V, Wood P, Weiss B, Treviño L. Ask Me 3: improving communication in a Hispanic pediatric outpatient practice. Am J Health Behav 2007; 31(Suppl 1): S115-21.

[21] Farrell M, Deuster L, Donovan J, Christopher S. Pediatric residents' use of jargon during counseling about newborn genetic screening results. Pediatrics 2008; 122(2): 243-9.

[22] Amer A, Fischer H. "Don't call me 'mom'": how parents want to be greeted by their pediatrician. Clin Pediatr 2009; $48: 720$. 